Revue Revue de l'histoire des religions

de Ihistoire des religions

2| 2007

Divination et révélation dans les mondes grec et romain

\title{
Divination et révélation dans les mondes grec et romain
}

Présentation

Nicole Belayche et Jörg Rüpke

\section{OpenEdition \\ Journals}

Édition électronique

URL : http://journals.openedition.org/rhr/5301

DOI : $10.4000 /$ rhr.5301

ISSN : 2105-2573

Éditeur

Armand Colin

\section{Édition imprimée}

Date de publication : 1 avril 2007

Pagination : 139-147

ISBN : 978-2200-92333-4

ISSN : 0035-1423

Référence électronique

Nicole Belayche et Jörg Rüpke, « Divination et révélation dans les mondes grec et romain », Revue de I'histoire des religions [En ligne], 2 | 2007, mis en ligne le 01 juin 2010, consulté le 22 septembre 2020. URL : http://journals.openedition.org/rhr/5301 ; DOI : https://doi.org/10.4000/rhr.5301 
NICOLE BELAYCHE

École pratique des Hautes Études, Paris

JÖRG RÜPKE

Université d'Erfurt

\section{Divination et révélation dans les mondes grec et romain Présentation}

En hommage à Jean-Pierre VERNANT

«Dans les sociétés où la divination ne revêt pas, comme dans la nôtre, le caractère d'un phénomène marginal, voire aberrant, où elle constitue une procédure normale, régulière, souvent même obligatoire $[\ldots] .{ }^{1}$

La divination est l'une des formes de la communication que les hommes établissent avec les dieux, c'est-à-dire une des composantes de la religion. Au sein des différents systèmes religieux, ce sont les hommes qui sont les initiateurs de la communication, même lorsque les dieux sont réputés envoyer différents signes ou ordres qui mettent l'homme en situation de répondre. Ces deux modalités étaient quotidiennes dans l'Antiquité classique. Elles sont toutes deux révélatrices d'une théologie et d'un énoncé de la conception du monde, quelle que soit la singularité des pratiques rituelles mises en œuvre pour gérer les messages demandés aux dieux ou envoyés par eux. Les formes divinatoires révèlent en outre le statut et la place que les hommes se donnent face aux dieux; elles font donc partie de

1. Jean-Pierre Vernant, « Paroles et signes muets », Divination et rationalité, Paris, 1974, p. 10. 
l'identité de l'homme dans une société donnée. Dans cette perspective, la divination est une des formes de définition de cette société et de ses membres. Elle ne relève pas uniquement de l'histoire des religions, mais implique les domaines politiques, sociaux et idéologiques.

Pourtant, la divination ne figure pas généralement dans les exposés sur la religion, du moins pas à une place prééminente. Le terme est utilisé pour décrire des techniques compliquées, d'aspect souvent exotique, mises en œuvre pour connaître la volonté du/des dieu/x et le futur. Depuis les associations spontanées à partir des motifs dessinés par l'huile dans de l'eau (lécanomancie) ou à partir de sons déclarés comme omineux (la « voix parlante/Aius Locutius » sur le Forum romain), jusqu' aux calculs astrologiques savants pratiqués par des mathematici professionnels, l'éventail des phénomènes est étendu et leurs manifestations sont bien souvent étudiées comme relevant d'un domaine spécifique. Animé d'un intérêt évident pour le marginal et l'inhabituel, Auguste Bouché-Leclercq en a répertorié les multiples pratiques dans un ouvrage en quatre volumes (plus de 1600 pages) : Histoire de la divination dans l'Antiquité. Cette étude analytique (par type de divination et par aire géographique) ne replace pas le phénomène divinatoire dans les conceptions et représentations grecque et romaine du monde. Cependant, sa richesse documentaire continue de la recommander comme une référence, ce dont témoigne sa réédition en $2003^{2}$. Depuis cette somme, la recherche a suivi plusieurs voies, munie des outils conceptuels et épistémologiques fournis par Divination et rationalité depuis $1974^{3}$. Cet ouvrage séminal s'est attaché à la nature des opérations intellectuelles à l'œuvre dans les pratiques divinatoires (la « rationalité ») et à la place du savoir divinatoire dans une société donnée. En construisant sur ces acquis, un groupe de recherche francoallemand (2004-2006) sur « La divination dans l'Antiquité (monde grec et empire romain) » a choisi d'explorer en quoi la divination était une modalité antique de l'action, évidente pour les futur et

2. Auguste Bouché-Leclercq, Histoire de la divination dans l'Antiquité, Paris, 1879-1882 (rééd. Grenoble, 2003, avec une Préface de Stella Georgoudi).

3. Jean-Pierre Vernant et al., Divination et rationalité, Paris, 1974. 
présent de l'homme, mais également mise en œuvre rétrospectivement pour le passé. C'est une fonction sociale dynamique, car elle agit comme une force de légitimation, autant dans un contexte collectif (celui de la cité, où le recours aux signes des dieux permettait de perpétuer ou de recomposer des équilibres) qu'individuel, et, chronologiquement, autant dans les périodes classiques que dans des siècles plus tardifs. Les cinq contributions de ce dossier reflètent le volet anthropologique des conclusions de ce groupe de recherche ${ }^{4}$.

Il n'est pas superflu de rappeler quelques repères thématiques et bibliographiques qui aideront à situer les questions abordées ici dans le contexte de la recherche récente ${ }^{5}$. Outre l'examen des pratiques, la divination en tant que technè mantikè a été prise en compte dans les études sur la réflexion théorique dans l'Antiquité, en se fondant plus spécialement sur le dialogue homonyme de Cicéron, le De divinatione ${ }^{6}$. Ce texte, qui expose de façon critique les principales positions de la philosophie grecque et hellénistique sur la divination, constitue un point de départ obligé pour toute investigation sur la pensée antique relative à la divination, comme en témoignent les commentaires fournis qu'il a suscités. Or, Cicéron, lui-même un spécialiste en divination en sa qualité d'augure du peuple romain, développe son argumentation à partir de deux points de vue opposés qui se partagent les livres I et II. Cette construction balancée a depuis longtemps posé au lecteur un délicat problème d'interprétation?

4. L'autre volet (social et politique) est publié dans les Cahiers du Centre Glotz, 16, 2005, «Divination et décision », Dominique Jaillard \& Katharina Waldner éd., p. 213-319. Le groupe de recherche (EPHE, Sciences religieuses, Paris [N. Belayche] et Université d'Erfurt, Allemagne [J. Rüpke]) a bénéficié du soutien d'un Programme d'Action Intégrée bi-national « Procope ».

5. Cf. Walter Burkert dir., « Mantik in Griechenland », Adriano Maggiani, « La divinazione in Etruria » et Nicole Belayche \& Jörg Rüpke dir., « Divination romaine », Thesaurus Cultus et Rituum Antiquorum (ThesCRA), III (2005), p. $1-104$.

6. Éd. \& comm. : Arthur S. Pease, M. Tulli Ciceronis De Divinatione libri duo, Cambridge (Mass.), 1920-1923 [rééd. Darmstadt, 1963] ; trad. fr. \& comm. : Gérard Freyburger \& John Scheid, Paris, 1992 ; trad. all. \& comm. : Christoph Schäublin, Marcus Tullius Cicero, Über die Wahrsagung, Darmstadt, 1991.

7. Cf. Mary Beard, "Cicero and Divination: The Formation of a Latin Discourse”, Journal of Roman Studies, 76 (1986), p. 33-46. 
L'usage politique de la divination, quand ce ne sont pas ses abus, a fourni un large champ de recherche. Du côté de la divination grecque, l'intérêt s'est porté vers les activités supra-régionales des oracles, ceux d'Apollon principalement - à Delphes, puis à Didymes et Claros en Ionie -, mais aussi ceux de Zeus à Dodone ${ }^{8}$. Du côté de la divination romaine, c'est l'augurat qui était le mode divinatoire le plus sollicité dans la mesure où il offrait des possibilités d'intervention directe dans l'action politique ${ }^{9}$. Dans le même registre, les prodiges, si nombreux dans l'historiographie antique, ont été analysés dans leurs dimensions littéraire, psychologique et politique ${ }^{10}$. Pour la période tardive, à partir du $\mathrm{III}^{\mathrm{e}}$ siècle, M.-Th. Fögen a mis en évidence l'emprise accrue du pouvoir politique jusque dans le domaine des devins professionnels ${ }^{11}$. En examinant plus spécialement le rôle de la divination dans les processus de décision, notre groupe de recherche franco-allemand a pu éclairer de façon significative le rôle joué par la divination dans l'action publique ${ }^{12}$.

Un autre axe d'interrogations a consisté en une analyse comparée des concepts de temps, de futur et de destin dans les diverses aires

8. Respectivement : Herbert W. Parke, The Oracles of Apollo in Asia Minor, Londres, 1985 et Aude Busine, Paroles d'Apollon. Pratiques et traditions oraculaires dans l'Antiquité tardive (II $-\mathrm{VI}^{e}$ siècles), Leyde-Boston, 2005 (RGRW 156) ; Herbert W. Parke, The Oracles of Zeus. Dodona - Olympia Ammon, Oxford, 1967 et Éric Lhote, Les lamelles oraculaires de Dodone, Genève (EPHE, Hautes études du monde gréco-romain 36), 2006.

9. Jerzy Linderski, "The Augural Law", Aufstieg und Niedergang der römischen Welt II 16, 3 (1986), p. 2146-2312 ; Leonhard A. Burckhardt, Politische Strategien der Optimaten in der späten römischen Republik, Stuttgart, 1988 ; Loretana de Libero, Obstruktion: Politische Praktiken im Senat und in der Volksversammlung der ausgehenden römischen Republik, 70-49 v. Chr., Stuttgart, 1992.

10. Raymond Bloch, La divination dans l'antiquité, Paris, 1984 ; Bruce MacBain, Prodigy and Expiation. A Study in Religion and Politics in Republican Rome, Bruxelles (coll. Latomus 177), 1982 ; Burkhard Gladigow, « Konkrete Angst und offene Furcht: Am Beispiel des Prodigienwesens in Rom », Heinrich von Stietencron éd., Angst und Gewalt: Ihre Präsenz und ihre Bewältigung in den Religionen, Düsseldorf, 1979, p. 61-77; Veit Rosenberger, Gezähmte Götter. Das Prodigienwesen der römischen Republik, Darmstadt, 1998.

11. Marie-Theres Fögen, Die Enteignung der Wahrsager: Studien zum kaiserlichen Wissensmonopol in der Spätantike, Francfort/Main, 1993.

12. Cf. supra n. 4 pour la publication. 
culturelles de l'Antiquité classique ${ }^{13}$. Mais les grandes entreprises comparatistes furent rares ${ }^{14}$. Même la recherche sur l'astrologie antique s'est surtout concentrée sur des problèmes techniques ${ }^{15}$; rares sont les tentatives pour resituer ces techniques dans le champ des savoirs contemporains et dans la vie quotidienne ${ }^{16}$.

Ce fut précisément l'un des points de départ de notre réflexion que de resituer les pratiques divinatoires dans la fabrique de la religion et, en confrontant différentes traditions de recherche, de sortir la « divination » du champ isolé, voire autonome, dans lequel elle était confinée pour l'analyser dans le contexte englobant de la communication religieuse. La communication religieuse est une forme de communication dans laquelle le(s) destinataire(s) ou interlocuteur(s) ne peuvent pas faire l'objet d'une vérification empirique. En conséquence, toute tentative de communication avec l'invisible suppose de le construire, de définir l'inconnaissable, de rendre les pouvoirs surnaturels visibles et audibles. Les multiples stratégies rituelles visent, donc, à intensifier, à matérialiser, parfois à monumentaliser, l'effort humain de communication, ce qui ne peut se faire sans spécialistes et sans délimiter des lieux et des temps propres. L'envoi de messages par les hommes utilise des paroles, des objets ou des êtres qui, par l'effet de la formalisation rituelle de la cérémonie, passent du statut de prière verbale ou d'offrande à celui de media capables d'atteindre le(s) interlocuteur(s). À l'inverse, les pratiques rassemblées sous le terme de « divination » donnent le premier rôle à l'autre partenaire - divin - de la communication. Elles doivent matérialiser sa réponse, ce qui fait attestation de sa présence, mais ce qui n'est pas sans risques, par exemple celui de l'incompréhension.

13. Raymond Bloch, La divination. Essai sur l'avenir et son imaginaire, Paris, 1991.

14. Cf. André Caquot \& Marcel Leibovici, La divination, Paris, 1968 et supra n. 3 .

15. Par ex. Tamsyn Barton, Ancient Astrology, Londres-New York, 1994.

16. Par ex. Sven Eriksson, Wochentagsgötter, Mond und Tierkreis: Laienastrologie in der römischen Kaiserzeit, Stockholm, 1956 et Kocku von Stuckrad, Das Ringen um die Astrologie: jüdische und christliche Beiträge zum antiken Zeitverständnis, Berlin-New York, 2000. 
Autant que faire se peut, ces pratiques doivent, donc, dissimuler la nature véritable de cette communication - construite et unilatérale.

Au plan théologique, désigner cet événement comme une « révélation» revient à braquer les feux sur l'agent divin. Dans les occasions divinatoires - qu'elles soient suscitées par une institution de consultation ou « subies » dans les rêves ou les songes éveillés -, la divinité se donne à lire ou à entendre, sinon toujours à voir. On a parfois tendance à réserver le concept de révélation aux trois religions issues d'Abraham, qualifiées précisément de « révélées » parce que l'épiphanie divine est réputée avoir transmis un message qui fut mis pour partie par écrit dans un texte devenu canonique (la Bible et le Coran). Un tel accent sur une manifestation divine, porteuse de message, à contenu rituel ou conceptuel - donc théologique dans les deux cas - existe pareillement dans la représentation romaine, qui plus est rapporté aux temps les plus anciens de Rome ${ }^{17}$. Au plan anthropologique, l'usage du concept de « révélation » permet de rendre compte de déclarations spécifiques ou occasionnelles gratifiées d'un statut d'autorité dans une communauté humaine.

À première vue - c'est du moins la perspective qui a guidé une bonne part de la recherche antérieure -, la divination antique apparaît comme un mode passif d'accès à une connaissance détenue par les dieux, donc comme une soumission à la révélation du futur :

« une connaissance d'une nature spéciale, plus ou moins directe, plus ou moins complète, mais toujours obtenue par voie de révélation surnaturelle, avec ou sans le concours du raisonnement. La divination a pour domaine tout ce que l'esprit humain ne peut connaître par ses seules forces : en premier lieu l'avenir, en tant qu'il échappe à la prévision rationnelle, puis le passé et le présent, dans ce qu'ils ont d'inaccessible à l'investigation ordinaire ${ }^{18}$.

Les mortels sont soumis à la volonté des dieux et font de leur mieux pour la pénétrer. Différentes techniques peuvent se compléter, voire

17. Cf. Ovide, Fastes 3, 327-348 (la négociation entre Jupiter et Numa pour procurer les foudres). Le déroulement narratif de l'apparition de Jupiter présente plusieurs similitudes avec celui de l'apparition de YHWH sur le Sinaï (Exode 3, 2-6).

18. A. Bouché-Leclercq, op. cit., I, p. 7. Pour cet auteur, la divination est « contemplative » et la magie « active ». 
se concurrencer, afin d'identifier et interpréter les signes signifiants, c'est-à-dire porteurs de messages divins dans la vie quotidienne, ou afin d'offrir aux divinités des occasions d'exprimer leur volonté de la façon la plus claire possible. Lorsque les dieux sont déclarés manifester leur pouvoir à travers les signa qu'ils envoient pour prévenir les hommes d'un manquement ou d'un dysfonctionnement, des rituels, oraculaires (la consultation de l'oracle de Delphes par ex.) ou divinatoires (la consultation des livres sibyllins) permettent d'affronter cette irruption du sacré.

Nous avons déjà signalé en quoi cette fiction de passivité était importante pour les contemporains ; aussi insistent-ils sur l'initiative divine ou sur son caractère arbitraire. La conséquence en est que l'action humaine (ou le refus d'agir) qui s'appuie sur la volonté divine ainsi établie est de facto légitimée. La parole des dieux apparaît comme une forme d'expression de décisions humaines qu'elle investit de légitimité, donc d'autorité. Cela permet de comprendre l'évolution de l'attitude des pouvoirs publics face à ces pratiques rituelles. Dans la Rome républicaine, les membres de l'élite politique remplissaient les fonctions de spécialistes en divination (les augures) et employaient des techniques (l'observation du vol des oiseaux ou de l'appétit des poulets sacrés) qui étaient facilement manipulables, ou rendues fictives puisque la déclaration de l'observation suffisait à faire office de consultation. En Grèce, les grands oracles suprarégionaux, qui combinaient des composantes de hasard avec des procédures sophistiquées d'interprétation/d'exègèsis, affichaient une image de rationalité extérieure sans être tenus par des règles contraignantes. En revanche, la multiplication d'oracles écrits, de prophètes charismatiques, ou de signes envoyés directement par les dieux sans passer par les spécialistes patentés de la communication (prêtres ou prophètes institutionnels) pouvait introduire des manifestations divines non conformes au cours des actes ou des valeurs portés par la société dominante et son élite.

La conception (emic comme etic) d'une divination comme un simple mode passif d'accès à un savoir envoyé par les dieux est manifestement inadéquate pour l'époque impériale. Le processus de consultation apparaît de plus en plus comme une modalité active 
pour changer le futur. Dès le dernier siècle de la République, secoué par les guerres civiles, puis à partir du règne d'Auguste, les consultations divinatoires, surtout lorsqu'elles concernaient des personnes publiques, pouvaient apparaître comme un moyen d'orienter le futur. De façon récurrente sous tous les règnes, la législation s'est efforcée de prévenir ou de punir les risques subversifs des consultations sur l'avenir de l'État, la vie des princes, voire des maîtres d'esclaves. À époque tardive, au plan public, elle est devenue ce que M.-Th. Fögen a appelé « un monopole du savoir par l'Empereur ${ }^{19}$. La conception nouvelle du pouvoir impérial recelait en elle-même une modification plus profonde, selon laquelle la compétence religieuse ne résidait plus automatiquement chez les membres de l'élite sociale et politique. Dans un certain nombre de contextes, l'autorité religieuse devint une qualité fondée sur des critères propres. En réaction, les autorités politiques traditionnelles brandirent des accusations de « sorcellerie » ou de «magie ». Avec le débat entre paganisme et christianisme et l'évolution du pouvoir impérial vers un «dominat", les auteurs chrétiens, comme la législation tardive, ont usé de l'argument pour discréditer toutes les pratiques païennes comme « magiques ».

Au plan cognitif, enfin, l'idée selon laquelle les pratiques divinatoires puissent opérer comme un pouvoir rituel capable d'agir sur le monde repose sur la croyance en une sympathie entre nature et surnature et, donc, en une réciprocité entre les deux mondes, à condition d'accomplir les bons gestes et de proférer les bonnes formules. Cette croyance est une condition nécessaire pour de nombreuses techniques. Sous la République déjà, le choix des protagonistes rituels (celui qui prononcera la prière publique) ou politiques (la première [dite prérogative] unité qui ira voter) était considéré comme un gage important pour la réussite de l'action. La recherche antérieure a généralement identifié ces croyances et pratiques comme « magiques », sans distinguer suffisamment les systématisations anciennes et modernes.

En s'attachant à des contextes culturels et historiques précis, les contributions de ce dossier invitent à un parcours à travers les

19. Op. cit. n. 11. 
dimensions cognitive et performative de la divination. Ouvert par Dominique Jaillard («Plutarque et la divination : la piété d'un prêtre philosophe »), ce parcours part de l'homme de Chéronée, car, comme Cicéron, il allie en sa personne le praticien public et l'exégète, mais, à la différence de l'augure romain, sa conceptualisation de la pratique divinatoire vient étayer l'attitude ritualiste. Les quatre contributions qui suivent ouvrent autant de portes sur les articulations établies entre le champ divinatoire et d'autres canaux possibles de paroles d'autorité. Nicole Belayche ("Les dieux "nomothètes". Oracles et prescriptions religieuses à l'époque romaine impériale ») s'interroge sur l'éventuelle spécificité des normes révélées par un dieu et replace les oracles «théologiques » d'époque tardive dans l'ensemble contemporain de toutes les productions des sanctuaires oraculaires. Se tournant vers le christianisme en train de délimiter ses frontières et de se donner des structures internes, Katharina Waldner («Les martyrs comme prophètes. Divination et martyre dans le discours chrétien des $\mathrm{I}^{\mathrm{er}}$ et $\mathrm{II}^{\mathrm{e}}$ siècles ») examine le rôle attribué à la qualité prophétique dans la figure émergeante du martyr et l'interprète au regard du contexte culturel de la Seconde sophistique. Opérant une indispensable remontée chronologique, Marcello Carastro ( Quand Tirésias devint un mágos. Divination et magie en Grèce ancienne, $V^{\mathrm{e}}-\mathrm{IV}$ siècles avant notre ère ») établit les bases idéologiques et épistémologiques sur lesquelles fut construit le couple antithétique religion et magie qui servira d'outil répressif à l'époque impériale. Dans ce même champ du statut attribué au savoir, Blossom Stefaniw (« Reading Revelation: Allegorical Exegesis in Late Antique Alexandria ») ouvre une piste problématique autre en analysant, dans le milieu multiconfessionnel de l'Alexandrie tardive, comment l'exégèse allégorique reposait sur la conviction de l'existence d'une révélation dans tous les textes de la tradition, classique autant que biblique.

n.belayche@wanadoo.fr joerg.ruepke@uni-erfurt.de 